\title{
Zur Chemotherapie der Gonorrhö
}

Nach den großen Erfolgen der von Ehrlich geschaffenen Chemotherapie der Syphilis, die als Bonner maximales Kursystem in mehr als 25 Jahren Aus-gezeichnetes zur Niederringung dieser Welfcplage geleistet hat, und nach der Aufklärung der vierten Geschlechtskrankheít, des Lymphogranuloma inguinale, dessen Erreger als Gramdozoon erkannt ward, blieb die wirksame Bekämp-fung der Gonorrhö das wichtigste Problem auf dem Gebiet der venerisehen Erkrankungen. Ausgehend von dem besonders in USA. bei Harnleiden ge-rühmten Prontosü, das, einfach innerlich gegeben, auf Streptokokken günstig einwirkt, hat G. Domagk (I. G. Laboratorium Elberfeld) nun neue Mittel ge-funden, die nach einer ersten Mitteilung von 0. Grütz aus der Bonner Klinik (Mü. med. Wschr., 1937, 1201) und einer zweiten von R. Felke in Wiesbaden (D. med. Wschr., 1937, 1393) die Chemotherapie des Trippers als grundsätzlich errungen und aussichtsreich erscheinen lassen. Beide Kliniker haben mit DB 87 und DB $90-$ letzteres soil üliron genannt werden - in Serien von wenigen (zirka 7) Tagen in etwa zwei Drittel der Fälle bei Go. der Prau und des Kindes nach Darreichung von Tabletten (4-7 Tabletten pro die, bis zu 3-5 g) Ausheilung gesehen. Durch Kombination mit den wohlbewährten Jawe $1 / 8$ chen Spülungen mit Kal. perm, hat Felke auch bei Männern Günstiges erreicht. Die Vermeidung von anfangs häufigen Nebenerscheinungen wird hoffentlich bald so weit gelingen, daß das Verfahren für die Praxis reif wird. Mögen weitere Erfolge, die Schreus, Memmesheimer u. a. auch bei Vulvo-vaginitis infantum bestätigen, den heißen Wunsch der Ärzte, dessen Erreichung sozialhygienisch, bevölkerungspolitisch und ökonomisch so dringlich und wichtig ist, bald noch vollkommener erfüllen und die deutsche Forschung so-wie die Bonner Hautklinik auch auf diesem Gebiet führend bleiben. E. H. 